This is an electronic reprint of the original article. This reprint may differ from the original in pagination and typographic detail.

Author(s): Repin, Sergey

Title: $\quad$ Localized forms of the LBB condition and a posteriori estimates for incompressible media problems

Year: $\quad 2018$

Version:

Please cite the original version:

Repin, S. (2018). Localized forms of the LBB condition and a posteriori estimates for incompressible media problems. Mathematics and Computers in Simulation, 145, 156-170. https://doi.org/10.1016/j.matcom.2016.05.004

All material supplied via JYX is protected by copyright and other intellectual property rights, and duplication or sale of all or part of any of the repository collections is not permitted, except that material may be duplicated by you for your research use or educational purposes in electronic or print form. You must obtain permission for any other use. Electronic or print copies may not be offered, whether for sale or otherwise to anyone who is not an authorised user. 


\section{Accepted Manuscript}

Localized forms of the LBB condition and a posteriori estimates for incompressible media problems

S. Repin

PII: $\quad$ S0378-4754(16)30081-7

DOI: $\quad$ http://dx.doi.org/10.1016/j.matcom.2016.05.004

Reference: $\quad$ MATCOM 4325

To appear in: Mathematics and Computers in Simulation

Received date: 11 November 2014

Revised date: 22 July 2015

Accepted date: 26 May 2016

Please cite this article as: S. Repin, Localized forms of the LBB condition and a posteriori estimates for incompressible media problems, Math. Comput. Simulation (2016), http://dx.doi.org/10.1016/j.matcom.2016.05.004

This is a PDF file of an unedited manuscript that has been accepted for publication. As a service to our customers we are providing this early version of the manuscript. The manuscript will undergo copyediting, typesetting, and review of the resulting proof before it is published in its final form. Please note that during the production process errors may be discovered which could affect the content, and all legal disclaimers that apply to the journal pertain. 


\title{
Localized forms of the LBB condition and a posteriori estimates for incompressible media problems
}

\author{
S. Repin \\ St. Petersburg Department of V.A.Steklov Institute of Mathematics, 191023, \\ Fontanka 27, Sankt-Petersburg, Russia \\ and University of Jyväskylä, P.O.Box 35, FI-40014, Jyväskylä, Finland
}

\begin{abstract}
The inf-sup (or LBB) condition plays a crucial role in analysis of viscous flow problems and other problems related to incompressible media. In this paper, we deduce localized forms of this condition that contain a collection of local constants associated with subdomains instead of one global constant for the whole domain. Localized forms of the LBB inequality imply estimates of the distance to the set of divergence free fields. We use them and deduce fully computable bounds of the distance between approximate and exact solutions of boundary value problems arising in the theory of viscous incompressible fluids. The estimates are valid for approximations, which satisfy the incompressibility condition only in a very weak (integral) form. Another important question considered in the paper is how to select a proper measures that should be used in error analysis. We show that such a measure is dictated by the respective error identity and discuss properties of the measure for the Stokes, Oseen, and Navier-Stokes problems.
\end{abstract}

Key words: Incompressible viscous fluids, LBB condition, a posteriori error estimates. 65N15, 76D07, 35Q30.

\section{Introduction}

Evolution of an incompressible fluid occupying a bounded Lipschitz domain $\Omega \in \mathbb{R}^{d}(d=2,3)$ is described by the system 


$$
\begin{aligned}
& \frac{\partial u}{\partial t}+u_{i} \frac{\partial u}{\partial x_{i}}-\operatorname{Div} \sigma=f, \quad(x, t) \in Q_{T}:=\Omega \times(0, T), \\
& \operatorname{div} u=0 \\
& \sigma=-p \mathbb{I}+\sigma_{d},
\end{aligned}
$$

where $u(x, t), p(x, t)$, and $\sigma(x, t)$ are the velocity (vector function), pressure (scalar function), and stress (tensor function), respectively. Next, $\mathbb{I}$ is the unit element of the space $\mathbb{M}_{s}^{d \times d}$ containing symmetric $d \times d$ matrices, the symbol Div is used to denote the divergence of tensor functions, and div denotes the divergence of vector fields. We assume that (1)-(3) describes a media with a constant density (for simplicity the density is set to one). The deviatoric part of the stress is subject to a potential type relation

$$
\sigma_{d} \in \partial \mathcal{W}(\varepsilon)
$$

where $\mathcal{W}$ is a convex functional (dissipative potential). Bingham type potentials $\mathcal{W}(\varepsilon)=\mu|\varepsilon|^{\alpha}+k_{*}|\varepsilon|$ describe a wide spectrum of viscous fluids. Here $\mu$ and $k_{*}$ are positive constants related to viscous and plastic properties of fluids, respectively, and $\alpha>1$ is the energy growth parameter.

The equations (1)-(3) represent the balance law, the incompressibility condition, and the material (constitutive) law, respectively, and the potential $\mathcal{W}$ is usually presented in terms of the small strain tensor $\varepsilon$. The system is supplied with initial and boundary conditions

$$
\begin{aligned}
u(x, t) & =g & & (x, t) \in \partial_{1} \Omega \times(0,+\infty), \\
\sigma n & =F & & (x, t) \in \partial_{2} \Omega \times(0,+\infty), \\
u(x, 0) & =\varphi(x) & & x \in \Omega .
\end{aligned}
$$

It is assumed that $g$ and $\varphi$ are sufficiently regular for a proper definition of the respective traces and satisfy the divergence free condition.

An important class of Newtonian fluids is related to the parameters $\alpha=2$ and $k_{*}=0$. In this case, $\sigma_{d}=\frac{\mu}{2}\left(\nabla u+(\nabla u)^{T}\right)$ and (1) is presented by the Navier-Stokes system

$$
\frac{\partial u}{\partial t}+\operatorname{Div}(u \otimes u)-\mu \triangle u=f+\nabla p
$$

It is known that for sufficiently regular solenoidal $\varphi(x)$ there exists a weak Leray-Hopf solution. For $d=2$, existence of a unique solution has been proved by O. Ladyzhenskaya (see (18)) but similar result for the case $d=3$ is not yet proved (see, e.g., discussions in $(14 ; 16 ; 35)$ ). However, discrete (semidiscrete) analogs of the Navie-Stokes equation are actively used in the mathematical modeling.

From the computational point of view, exact satisfaction of the divergence 
free (incompressibility) condition (2) may lead to serious difficulties. Typically, numerical methods are based on variational or minimax settings and mixed velocity-pressure or velocity-stress-pressure settings and use finite dimensional spaces subject to discrete inf-sup conditions (see, e.g., $(3 ; 11 ; 15 ; 35)$ ) and in the majority of cases numerical solutions satisfy (2) only approximately. In this paper, we suggest a method of measuring errors that arise due to violation of the incompressibility condition. These results yield computable majorants of the distance to the exact solution for a wide set of viscous flow problems.

The main idea can be explained with the paradigm of the stationary Stokes problem: find $u$ and $p$ such that $u=0$ on $\partial \Omega$ and in $\Omega$

$$
\begin{aligned}
\operatorname{div} u & =0, \\
-\operatorname{Div} \sigma & =f, \\
\sigma & =\mu \nabla u-p \mathbb{I} .
\end{aligned}
$$

First, let us fix notation. Henceforth, $W^{1, \alpha}$ denotes the standard Sobolev space of scalar and vector valued functions having first generalized derivatives summable with the power $\alpha$. The respective norms are denoted by $\|\cdot\|_{1, \alpha}$. If $\alpha=2$, then we use a simplified notation $\|\cdot\|$ for $L^{2}$ norms of scalar, vector, and tensor valued functions. $W_{0}^{1, \alpha}$ denotes the subspace of $W^{1, \alpha}$ containing functions vanishing on the boundary and $S_{0}^{1,2}\left(\Omega, \mathbb{R}^{d}\right)$ denotes the closure (with respect to $W^{1,2}$ - norm) of smooth divergence free fields with compact supports in $\Omega$. Test functions in $S_{0}^{1,2}\left(\Omega, \mathbb{R}^{d}\right)$ are denoted by $\stackrel{\circ}{v}$ (or $\stackrel{\circ}{w}$ ). Also, we use the spaces

$$
\widetilde{L}^{2}(\Omega):=\left\{q \in L^{2}(\Omega) \mid\{q\}_{\Omega}:=(\text { meas } \Omega)^{-1} \int_{\Omega} q d x=0\right\}
$$

and

$$
H(\Omega, \operatorname{Div}):=\left\{\tau \in L^{2}\left(\Omega, \mathbb{M}^{d \times d}\right), \operatorname{Div} \tau \in L^{2}\left(\Omega, \mathbb{R}^{d}\right)\right\} .
$$

First, we recall the following result (see $(26 ; 27)$ ).

Theorem 1 Let $(u, p)$ be a weak solution to the Stokes problem (9)-(11). For any $v \in S_{0}^{1,2}\left(\Omega, \mathbb{R}^{d}\right), q \in \widetilde{L}^{2}(\Omega), \tau \in H(\Omega, \mathrm{Div})$,

$$
\|\mu \nabla(u-v)\| \leq\|\tau-\mu \nabla v+q \mathbb{I}\|+C_{F \Omega}\|\operatorname{Div} \tau+f\|=: M(v, q, \tau),
$$

where $C_{F \Omega}$ is a constant in the Friedrichs inequality $\|\psi\| \leq C_{F \Omega}\|\nabla \psi\|$ for any $\psi \in W_{0}^{1,2}(\Omega)$. Moreover, the majorant $M(v, q, \tau)$ vanishes if and only if $v=u, q=p$, and $\tau=\sigma$.

Similar estimates have been derived for the generalized Stokes problem (32), Stokes problem in the velocity-vorticity formulation (21), NonNewtonian fluids $(12 ; 13)$, Oseen and generalized Oseen problems $(28 ; 30)$. 
Theorem 1 shows that the Stokes problem is equivalent to the following variational Problem: find $u \in S_{0}^{1,2}\left(\Omega, \mathbb{R}^{d}\right), p \in \widetilde{L}^{2}(\Omega)$, and $\sigma \in H(\Omega$, Div) such that

$$
\inf _{\substack{v \in S_{0}^{1,2}\left(\Omega, \mathbb{R}^{d}\right), \tau \in H(\Omega, \text { Div }), q \in \widetilde{L}^{2}(\Omega)}} M(v, q, \tau)=M(u, p, \sigma)=0 .
$$

In principle, this problem can be used for finding $u, p$, and $\sigma$ by means of direct minimization of the majorant $M(v, q, \tau)$. However, this method requires approximations of the velocity in $S_{0}^{1,2}$ what generates well known technical difficulties (especially in 3D case). Therefore, we wish to extend the space $S_{0}^{1,2}\left(\Omega, \mathbb{R}^{d}\right)$ in Problem $(13)$ to a wider space $W_{0}^{1,2}\left(\Omega, \mathbb{R}^{d}\right)$. For this purpose, we need a projection type estimate

$$
\inf _{\stackrel{\circ}{v \in S_{0}^{1,2}\left(\Omega, \mathbb{R}^{d}\right)}}\|\nabla(v-\stackrel{\circ}{v})\| \leq \Pi_{S_{0}^{1,2}}(v)
$$

where $\Pi_{S_{0}^{1,2}}(v)$ is a computable functional. By (12) and (14), we obtain

$$
\begin{aligned}
\underline{\mu\|\nabla(u-v)\|} & \leq \mu\|\nabla(u-\stackrel{\circ}{v})\|+\mu\|\nabla(\stackrel{\circ}{v}-v)\| \\
& \leq\|\mu \nabla \stackrel{\circ}{v}-\tau-q \mathbb{I}\|+C_{F \Omega}\|\operatorname{div} \tau+f\|+\mu\|\nabla(\stackrel{\circ}{v}-v)\| \\
& \leq\|\mu \nabla v-\tau-q \mathbb{I}\|+C_{F \Omega}\|\operatorname{div} \tau+f\|+2 \mu\|\nabla(\stackrel{\circ}{v}-v)\| \\
& \leq\|\mu \nabla v-\tau-q \mathbb{I}\|+C_{F \Omega}\|\operatorname{div} \tau+f\|+2 \mu \Pi_{S_{0}^{1,2}}(v),
\end{aligned}
$$

where $v \in W_{0}^{1,2}\left(\Omega, \mathbb{R}^{d}\right), \tau \in H(\Omega$, Div $)$, and $q \in \widetilde{L}^{2}(\Omega)$. We see that the right hand side of (15) contains three terms associated with the equations (9), (10), and (11). In other words, being supplied with proper weights the least squares type complex in the right hand side majorates the error and vanishes if and only if $v, \tau, q$ coincide with the exact velocity, stress, and pressure. It is known that $\Pi_{S_{0}^{1,2}}(v)$ can be expressed via $\|\operatorname{div} v\|$ (see $(26 ; 27)$; similar results also hold for $L^{\alpha}$ based spaces (31)).

The relations (13) and (15) suggest least square type functionals (with the weight factors that provide proper scaling of different terms) that should be used for getting numerical approximations of the Stokes problem. For example, we can minimize the functional

$$
F\left(v_{h}, \tau_{h}\right):=\left\|\left(\mu \nabla v_{h}-\tau_{h}\right)^{D}\right\|^{2}+C_{F \Omega}^{2}\left\|\operatorname{div} \tau_{h}+f\right\|^{2}+4 \mu^{2} \Pi_{S_{0}^{1,2}}^{2}\left(v_{h}\right)
$$

over $v_{h} \in V_{h}$ and $\tau_{h} \in Z_{h}$, where $V_{h}$ and $Z_{h}$ are certain finite dimensional subspaces of $W_{0}^{1,2}\left(\Omega, \mathbb{R}^{d}\right)$ and $H(\Omega$, Div $)$, respectively, which are generated by a mesh having cells of the size $h$. To obtain (16), we set $q_{h}=\frac{1}{d}\left(\mu \operatorname{div} v_{h}-\operatorname{tr} \tau_{h}\right)$. 
The the first term of the majorant (15) contains only deviatoric part of the tensor $\mu \nabla v_{h}-\tau_{h}$. From (15) and the inequality $(a+b+c)^{2} \leq 3\left(a^{2}+b^{2}+c^{2}\right)$, it follows that

$$
F\left(v_{h}, \tau_{h}\right) \geq \frac{\mu^{2}}{3}\left\|\nabla\left(u-v_{h}\right)\right\|^{2} .
$$

Hence global minimisation of $F\left(v_{h}, \tau_{h}\right)$ is a stable process, which generates monotonically decreasing values of the functional and explicitly controls the errors. It can be performed by different methods (direct minimisation of reduction to a system of algebraic equations and using techniques developed for the least squares mixed finite element methods, see, e.g., (4)). Optimisation of minimization procedures leads to interesting algebraic problems, which are beyond the framework of our analysis. We only mention that for simpler diffusion type equations they are discussed in, e.g., (36), where a version of the multigrid method was suggested for efficient minimization of similar functionals.

The outline of the paper is as follows. In Section 2, we present a unified approach to a posteriori error estimation for models of viscous incompressible fluids. In the first part 2.1, we establish the error identity (21), which holds for the quantities available in computations and a proper error measure (in general, a nonlinear pseudometric). This identity shows that the measure cannot be selected arbitrary and is foreordained by the nature of the problem.

The part 2.2, is devoted to fully computable bounds of the distance between exact solutions and approximations of the considered class of problems (which includes the Stokes, Oseen, and Navier-Stokes equations as particular cases). These estimates can be useful not only for the error control. They generate nonnegative least squares type functionals, which vanish if and only if the approximations entering the functional coincide with the exact stress, pressure, and velocity fields. The structure of the functionals is such that exact satisfaction of the divergence free condition is not required. Instead the majorant contains the term $\kappa\|\operatorname{div} v\|$ with the finite penalty multiplier $\kappa$, which depends on $\Omega$ only.

In Section 3, we show that $\kappa(\Omega)$ coincides with the constant in the BabuškaAziz-Ladyzhenskaya-Solonnikov inequality and discuss some cases where bounds of this constant are known. Regrettably, the amount of these cases is very limited (e.g., for polyhedral domains in $\mathbb{R}^{3}$ up to now it is not known how to find $\kappa(\Omega)$ or sharp majorants of this constant). Results exposed in Section 4 shows a way to bypass these difficulties. If $\Omega$ is decomposed into a collection of "simple" subdomains $\Omega_{i}$ (for which $\kappa\left(\Omega_{i}\right)$ or the respective majorants are known), then the distance to the set of divergence free fields can be estimated. Using this result, we obtain a fully computable majorant of the distance between $u$ and $v$, which is valid for non divergence free fields. This majorant and can be used for guaranteed error control and also for various numerical minimization 
procedures generating approximations of solutions to incompressible media problems.

\section{Estimates of the distance to the exact solution}

\subsection{Measure of the distance}

First, we introduce and justify a measure to be used for estimating the accuracy of approximate solutions to (1)-(3). In order to simplify the discussion and present the conception in the most transparent way, we begin with the stationary version of (1)-(3) supplied with the Dirichlet boundary condition $u(x)=g$ on the boundary $\partial \Omega$ (we assume that $\operatorname{div} g=0$ ). Generalizations are discussed in section 2.4. We define the tensor

$$
\pi(u):=\sigma_{d}(u)-\varpi(u) .
$$

By selecting $\varpi(u)$ in deferent ways, we can analyse various models of viscous fluids within the framework of a unified scheme. At this point we do not confine ourselves with a particular choice of $\varpi(u)$ assuming only that it is defined such that the integrand in the left hand side of (18) is a summable function for any test function $\stackrel{\circ}{w}$. Concrete forms of $\varpi(u)$ are considered in Section 2.3.

Henceforth, $S_{0}\left(\Omega, \mathbb{R}^{d}\right)$ denotes a space of solenoidal functions, which properties are induced by the energy space $V_{0}\left(\Omega, \mathbb{R}^{d}\right)$ containing $S_{0}\left(\Omega, \mathbb{R}^{d}\right)$ (e.g., for problems with Bingham type dissipative potentials, $V_{0}\left(\Omega, \mathbb{R}^{d}\right)$ is the Sobolev space $W_{0}^{1, \alpha}\left(\Omega, \mathbb{R}^{d}\right)(\alpha>1)$ and for problems with Newtonian potentials $S_{0}\left(\Omega, \mathbb{R}^{d}\right)=$ $\left.S_{0}^{1,2}\left(\Omega, \mathbb{R}^{d}\right)\right)$. The norm of $S_{0}$ is denoted by $\|\cdot\|_{S_{0}}$.

The generalized solution $u \in S_{0}\left(\Omega, \mathbb{R}^{d}\right)+g$ is defined by the integral identity

$$
\int_{\Omega} \pi(u): \nabla \stackrel{\circ}{w} d x=\int_{\Omega} f \cdot \stackrel{\circ}{w} d x \quad \forall \stackrel{\circ}{w} \in S_{0}\left(\Omega, \mathbb{R}^{d}\right) .
$$

In general, the source term $f$ is an element of the space $S_{0}^{*}$ conjugate to $S_{0}$. However, for the sake of simplicity we henceforth assume that it is a function integrable with some power greater than 1 .

For $v \in S_{0}\left(\Omega, \mathbb{R}^{d}\right)+g$, we introduce the functional

$$
\mathcal{L}_{v}(\stackrel{\circ}{w}):=\int_{\Omega}(f \cdot \stackrel{\circ}{w}-\pi(v): \nabla \stackrel{\circ}{w}) d x
$$

which is the residual of (18) generated by $v$ and computed on a test function 
$\stackrel{\circ}{w}$. The value of

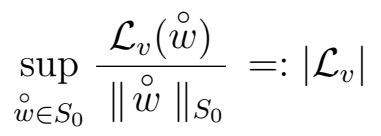

characterises the quality of $v$ considered as an approximation of the generalised solution $u$. For any $\widetilde{u} \in V_{0}+g$, we define the quantity

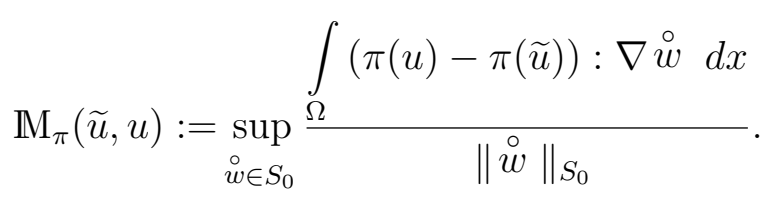

It is easy to see that $\mathrm{M}_{\pi}(\widetilde{u}, u)$ is nonnegative, symmetric, satisfies the triangle inequality, and vanishes if $\widetilde{u}=u$. Therefore, $\mathbb{M}_{\pi}(\widetilde{u}, u)$ can be viewed as a certain measure of the distance between $\widetilde{u}$ and the exact solution $u$.

In view of (18), we have

$$
\mathrm{M}_{\pi}(v, u)=\left|\mathcal{L}_{v}\right|
$$

Here $\left|\mathcal{L}_{v}\right|$ is a natural norm of the residual functional that contains all available information on the quality of the approximate solution $v$. Therefore, the error identity (21) shows that the conception of minimization of $\left|\mathcal{L}_{v}\right|$ (which is explicitly or implicitly adopted by all numerical methods) automatically induces a local topology at the neighbourhood of $u$ (which is defined by the pseudometric (20)). Substantially, this means that $\mathrm{M}_{\pi}(v, u)$ is the measure to be used in quantitative analysis of the considered class of problems (see also $(29 ; 30)$ for a discussion of close questions related to proper selection of error measures for various nonlinear models).

\subsection{Majorant of $\mathbb{M}_{\pi}(v, u)$}

Now, we are concerned with the functional $\mathcal{L}_{v}$. It can be decomposed into two physically meaningful parts by means of a suitable integral identity (see (28) for a consequent exposition).

Let $Y:=\nabla S_{0}\left(\Omega, \mathbb{R}^{d}\right)$ (i.e., $Y$ contains the tensor valued functions, which are gradients of all vector functions in $\left.S_{0}\right) . Y^{*}$ denotes the corresponding dual space. We introduce another pair of mutually conjugate Banach spaces $\mathcal{V}$ and $\mathcal{V}^{*}$ such that $V_{0} \subset \mathcal{V}, \mathcal{V}^{*} \subset S_{0}^{*}$. Let $\tau \in H_{\text {Div }}(\Omega):=\left\{\tau \in Y^{*} \mid \operatorname{Div} \tau \in \mathcal{V}^{*}\right\}$. Since

$$
\int_{\Omega}(\tau: \nabla \stackrel{\circ}{w}+\operatorname{Div} \tau \cdot \stackrel{\circ}{w}) d x=0 \quad \forall \stackrel{\circ}{w} \in S_{0}\left(\Omega, \mathbb{R}^{d}\right)
$$


we represent $\mathcal{L}_{v}(\stackrel{\circ}{w})$ in the form

$$
\mathcal{L}_{v}(\stackrel{\circ}{w})=\int_{\Omega}(f+\operatorname{Div} \tau) \cdot \stackrel{\circ}{w} d x+\int_{\Omega}(\tau-\pi(v)+q \mathbb{I}): \nabla \stackrel{\circ}{w} d x
$$

where $q$ is any scalar valued function such that $q \mathbb{I} \in Y^{*}$. In view of (19), we have

$$
\left|\mathcal{L}_{v}\right| \leq C_{1}(\Omega)\|f+\operatorname{Div} \tau\|_{\mathcal{V}^{*}}+C_{2}(\Omega)\|\tau-\pi(v)+q \mathbb{I}\|_{Y^{*}},
$$

where $C_{1}(\Omega)$ and $C_{2}(\Omega)$ are positive constants in the embedding type inequalities

$$
\begin{aligned}
\|\stackrel{\circ}{w}\|_{\mathcal{V}} & \leq C_{1}(\Omega)\|\stackrel{\circ}{w}\|_{S_{0}} \quad \forall \stackrel{\circ}{w} \in S_{0}\left(\Omega, \mathbb{R}^{d}\right), \\
\|\nabla \stackrel{\circ}{w}\|_{Y} & \leq C_{2}(\Omega)\|\stackrel{\circ}{w}\|_{S_{0}} .
\end{aligned}
$$

We recall (21) and arrive at the following general estimate:

$$
\mathrm{M}_{\pi}(v, u) \leq C_{1}(\Omega)\|f+\operatorname{Div} \tau\|_{\mathcal{V}^{*}}+C_{2}(\Omega)\|\tau-\pi(v)+q \mathbb{I}\|_{Y^{*}}
$$

The right hand side of (24) is the error majorant. For any $v \in S_{0}\left(\Omega, \mathbb{R}^{d}\right)+g$ and $\tau \in H_{\text {Div }}(\Omega)$ it majorates the measure $\mathbb{M}_{\pi}(v, u)$.

Now our goal is to modify (24) and deduce a guaranteed upper bound of $\mathrm{M}_{\pi}(v, u)$ valid for any $v \in V_{0}+g$. First, we note that

$$
\mathbb{M}_{\pi}(v, u) \leq \mathbb{M}_{\pi}(w, u)+\mathbb{M}_{\pi}(v, w)
$$

for any $w \in S_{0}\left(\Omega, \mathbb{R}^{d}\right)+g$. Since

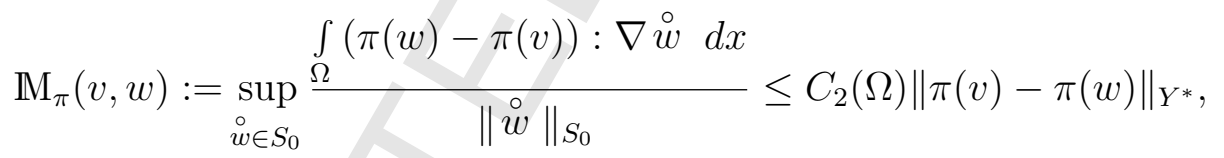

we find that

$$
\begin{aligned}
\mathbb{M}_{\pi}(v, u) \leq & \mathbb{M}_{\pi}(w, u)+\inf _{w \in S_{0}\left(\Omega, \mathbb{R}^{d}\right)+g} C_{2}(\Omega)\|\pi(v)-\pi(w)\|_{Y^{*}} \\
& \leq C_{1}(\Omega)\|f+\operatorname{Div} \tau\|_{\mathcal{V}^{*}}+C_{2}(\Omega)\|\tau-\pi(v)+q \mathbb{I}\|_{Y^{*}} \\
& +2 \inf _{w \in S_{0}\left(\Omega, \mathbb{R}^{d}\right)+g} C_{2}(\Omega)\|\pi(v)-\pi(w)\|_{Y^{*}} .
\end{aligned}
$$

For problems associated with Newtonian fluids, the last term in the right hand side of $(25)$ is estimated from above by $\Pi_{S_{0}^{1,2}}(v)$. 


\subsubsection{The Stokes problem.}

In this case, $S_{0}\left(\Omega, \mathbb{R}^{d}\right)=S_{0}^{1,2}\left(\Omega, \mathbb{R}^{d}\right), \sigma_{d}(v)=\pi(v)=\mu \nabla v$, the norm $\|w\|_{S_{0}}$ can be identified with $\|\nabla w\|, Y=Y^{*}=L^{2}\left(\Omega, \mathbb{M}^{d \times d}\right)$, and

$$
\mathrm{M}_{\pi}(u, v)=\|\mu \nabla(u-v)\| .
$$

We set $\mathcal{V}=L^{2}\left(\Omega, \mathbb{R}^{d}\right), V_{0}\left(\Omega, \mathbb{R}^{d}\right)=W_{0}^{1,2}\left(\Omega, \mathbb{R}^{d}\right), H_{\text {Div }}(\Omega)=H(\Omega$, Div $)$ and assume that $q \in \widetilde{L}^{2}(\Omega)$ (since $q$ is defined up to a constant we use this standard reduction of the admissible space for the pressure). Then, (22) and (23) hold with $C_{1}(\Omega)=C_{F \Omega}$ (Friedrihs' constant), $C_{2}(\Omega)=1$. If $v \in S_{0}\left(\Omega, \mathbb{R}^{d}\right)+g$, then (24) yields the estimate

$$
\mu\|\nabla(u-v)\| \leq C_{F \Omega}\|f+\operatorname{Div} \tau\|+\|\tau-\mu \nabla v+q \mathbb{I}\|,
$$

which coincides with (22).

Let $v \in W_{0}^{1,2}\left(\Omega, \mathbb{R}^{d}\right)$ and $w \in S_{0}^{1,2}\left(\Omega, \mathbb{R}^{d}\right)+g$. These functions can be represented in the form $v=g+v_{0}$ and $w=g+\stackrel{\circ}{w}$, where $\stackrel{\circ}{w} \in S_{0}^{1,2}\left(\Omega, \mathbb{R}^{d}\right)$ and $v_{0} \in W_{0}^{1,2}\left(\Omega, \mathbb{R}^{d}\right)$. Therefore, $\|\pi(v)-\pi(w)\|=\mu\left\|\nabla\left(v_{0}-\stackrel{\circ}{w}\right)\right\|$ and

$$
\mu\|\nabla(u-v)\| \leq\|\tau-\mu \nabla v+q \mathbb{I}\|+C_{F \Omega}\|\operatorname{Div} \tau+f\|+2 \mu \Pi_{S_{0}^{1,2}}\left(v_{0}\right) .
$$

In view of $(35), \Pi_{S_{0}^{1,2}}\left(v_{0}\right)=\kappa_{\Omega}\left\|\operatorname{div} v_{0}\right\|=\kappa_{\Omega}\|\operatorname{div} v\|$, and we arrive at the estimate (cf. $(26 ; 28)$ )

$$
\mu\|\nabla(u-v)\| \leq\|\tau-\mu \nabla v+q \mathbb{I}\|+C_{F \Omega}\|\operatorname{Div} \tau+f\|+2 \kappa_{\Omega}\|\operatorname{div} v\| .
$$

\subsubsection{The Oseen problem.}

For this problem, the spaces $S_{0}, \mathcal{V}$, and $Y$ are the same and (17) has the form $\pi(v)=\mu \nabla v-\mathrm{a} \otimes v$ (i.e., $\varsigma=\mathrm{a} \otimes v$ ). For any $w \in S_{0}^{1,2}$ we have

$$
\int_{\Omega} \varpi(w): \nabla w d x=-\frac{1}{2} \int_{\Omega} \mathrm{a} \cdot \nabla\left(|w|^{2}\right) d x=0 .
$$

Therefore,

$$
\mathrm{M}_{\pi}(\widetilde{u}, u) \geq \mu\|\nabla(u-\widetilde{u})\|
$$

and for any $v \in S_{0}^{1,2}\left(\Omega, \mathbb{R}^{d}\right)+g$ we obtain

$$
\mu\|\nabla(u-v)\| \leq C_{F \Omega}\|f+\operatorname{Div} \tau\|+\|\tau-\mu \nabla v+\mathrm{a} \otimes v+q \mathbb{I}\| .
$$


If $v=v_{0}+g$ and $v_{0}$ does not belong to $S_{0}^{1,2}\left(\Omega, \mathbb{R}^{d}\right)$, then we use $(25)$ and the estimate

$$
\|\pi(v)-\pi(w)\| \leq K(\mathrm{a})\|\nabla(v-w)\|=K(\mathrm{a})\left\|\nabla\left(v_{0}-\stackrel{\circ}{w}\right)\right\|,
$$

where $K(\mathrm{a})=C_{F \Omega}\|\mathrm{a}\|_{\infty, \Omega}+\mu$. Then, the estimate reads

$$
\begin{aligned}
& \mu\|\nabla(u-v)\| \\
& \quad \leq C_{F \Omega}\|f+\operatorname{Div} \tau\|+\|\tau-\mu \nabla v+\mathrm{a} \otimes v+q \mathbb{I}\|+2 K(\mathrm{a}) \Pi_{S_{0}^{1,2}}(v) .
\end{aligned}
$$

\subsubsection{The Navier-Stokes problem.}

Let $v \in S_{0}^{1,2}\left(\Omega, \mathbb{R}^{d}\right)+g$. For the Navier-Stokes problem $\pi(v)=\mu \nabla v-v \otimes v$. In this case, the estimate (24) has the form

$$
\mathbb{M}_{\pi}(v, u) \leq C_{F \Omega}\|f+\operatorname{Div} \tau\|+\|\tau-\mu \nabla v+v \otimes v+q \mathbb{I}\| .
$$

Such a simple lower bound of $\mathrm{M}_{\pi}(v, u)$ as in (27) does not take place. We can only prove (see (28)) that $\mathbb{M}_{\pi}(v, u)$ is bounded from below by $c\|\nabla(v-u)\|$ (where $c$ is a positive multiplier) provided that $\nabla v$ is sufficiently small. Moreover, may be in general $\mathbb{M}_{\pi}(v, u)$ is not a metric (actually, this question is related to the uniqueness of a weak solution to the Navier-Stokes equation).

Consider the estimate (25). It is easy to see that

$$
\|\pi(v)-\pi(w)\| \leq \mu\|\nabla(v-w)\|+\|v \otimes v-w \otimes w\|
$$

and $v \otimes v-w \otimes w==\left(v_{0}-\stackrel{\circ}{w}\right) \otimes v+v \otimes\left(v_{0}-\stackrel{\circ}{w}\right)-\left(v_{0}-\stackrel{\circ}{w}\right) \otimes\left(v_{0}-\stackrel{\circ}{w}\right)$. Therefore,

$$
\begin{aligned}
& \|\pi(v)-\pi(w)\| \\
& \qquad \mu\left\|\nabla\left(v_{0}-\stackrel{\circ}{w}\right)\right\|+2\left(\int_{\Omega}|v|^{2}\left|v_{0}-\stackrel{\circ}{w}\right|^{2} d x\right)^{1 / 2}+\left\|\left(v_{0}-\stackrel{\circ}{w}\right)^{2}\right\| \\
& \leq \mu\left\|\nabla\left(v_{0}-\stackrel{\circ}{w}\right)\right\|+2\|v\|_{4, \Omega}\left\|v_{0}-\stackrel{\circ}{w}\right\|_{4, \Omega}+\left\|v_{0}-\stackrel{\circ}{w}\right\|_{4, \Omega}^{2} .
\end{aligned}
$$

Since $W^{1,2}(\Omega)$ is embedded in $L^{4}(\Omega)$, there exists a constant $\varsigma(\Omega)$ such that

$$
\|\psi\|_{4, \Omega} \leq \varsigma(\Omega)\|\nabla \psi\| \quad \forall \psi \in \stackrel{\circ}{W}^{1,2}(\Omega)
$$

For $d=2$ we have the estimate $\|w\|_{4, \Omega} \leq \sqrt{2}\|w\|^{1 / 2}\|\nabla w\|^{1 / 2}$ and for $d=3$ $\|w\|_{4, \Omega} \leq \delta\|w\|^{1 / 4}\|\nabla w\|^{3 / 4}$ (where $\delta=\left(\frac{4}{3}\right)^{3 / 8}$ ) (see (18), Chapter 1). These 
estimates show that

$$
\varsigma(\Omega) \leq\left(2 C_{F}\right)^{1 / 2} \quad \text { for } d=2 \quad \text { and } \quad \varsigma(\Omega) \leq \delta\left(C_{F}\right)^{1 / 4} \quad \text { for } d=3 .
$$

Hence $\left\|v_{0}-\stackrel{\circ}{w}\right\|_{4, \Omega} \leq \varsigma(\Omega)\left\|\nabla\left(v_{0}-\stackrel{\circ}{w}\right)\right\|$ and

$$
\inf _{w \in S_{0}^{1,2}\left(\Omega, \mathbb{R}^{d}\right)+g}\|\pi(v)-\pi(w)\| \leq \Pi_{S_{0}^{1,2}}\left(v_{0}\right)\left(\mu+2 \varsigma(\Omega)\|v\|_{4, \Omega}^{2}+\varsigma^{2}(\Omega) \Pi_{S_{0}^{1,2}}\left(v_{0}\right)\right) .
$$

Since $\Pi_{S_{0}^{1,2}}\left(v_{0}\right)=\kappa_{\Omega}\|\operatorname{div} v\|$, we conclude that

$$
\inf _{w \in S_{0}^{1,2}\left(\Omega, \mathbb{R}^{d}\right)+g}\|\pi(v)-\pi(w)\| \leq \kappa_{\Omega} K(v)\|\operatorname{div} v\|,
$$

where $K(v)=\left(\mu+2 \varsigma(\Omega)\|v\|_{4, \Omega}^{2}+\varsigma^{2}(\Omega) \kappa_{\Omega}\|\operatorname{div} v\|\right)$. By (28) and (29), we obtain

$$
\mathbb{M}_{\pi}(v, u) \leq C_{F \Omega}\|f+\operatorname{Div} \tau\|+\|\tau-\mu \nabla v+v \otimes v+q \mathbb{I}\|+2 K(v) \kappa_{\Omega}\|\operatorname{div} v\|
$$

$\mathrm{M}_{\pi}(v, u)$ measures the distance between $v$ and a vector valued function $u$ that satisfy (in a weak sense) the Navier-Stokes equation and the prescribed boundary condition. Hence if the respective solution is not unique, then (30) shows that the measure $\mathbb{M}_{\pi}$ induced by $\mathcal{L}_{v}$ cannot be a metric. The same conclusion is true for the general estimate (25).

\subsection{Evolutionary problems}

We conclude this section with comments that extend above presented conception to evolutionary equations (1)-(3) considered in the space-time cylinder $Q_{T}$ with the initial and boundary conditions (5), (6), and (7). Let $v(x, t)$ be an approximation of $u(x, t)$ which belongs to the same functional class as $u(x, t)$ and satisfies the prescribed boundary conditions. The function $v(x, t)$ generates the functional

$$
\mathcal{L}_{v}(\stackrel{\circ}{w}):=\int_{Q_{T}}\left(f \cdot \stackrel{\circ}{w}-\pi(v): \nabla \stackrel{\circ}{w}-v_{t} \cdot \stackrel{\circ}{w}\right) d t d x .
$$

Here $v_{t}$ denotes the time derivative and it is assumed that $v(x, t)$ and the test function $\stackrel{\circ}{w}(x, t)$ is divergence free for any $t \in[0, T]$. Let $\|\stackrel{\circ}{w}\|_{S_{0}\left(Q_{T}\right)}$ be the norm in $S_{0}\left(Q_{T}\right):=L^{\gamma}\left((0, T), S_{0}\left(\Omega, \mathbb{R}^{d}\right)\right.$ ) (suitable value of $\gamma$ depends on properties of $\pi)$. Similarly to (19), the quantity

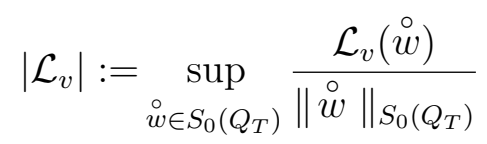


characterises the quality of $v(x, t)$ as an approximation of (1)-(4). It uses all the information available in numerical experiments. The basic error identity

$$
\mathrm{M}_{\pi}(v, u)=\left|\mathcal{L}_{v}\right|
$$

holds if the distance is defined by the measure

$$
\mathrm{M}_{\pi}(v, u):=\sup _{\stackrel{\dot{w}}{w} \in S_{0}\left(Q_{T}\right)} \frac{\int_{Q_{T}}\left((\pi(u)-\pi(v)): \nabla \stackrel{\circ}{w}+\left(u_{t}-v_{t}\right) \cdot \stackrel{\circ}{w}\right) d t d x}{\|\stackrel{\circ}{w}\|_{S_{0}\left(Q_{T}\right)}} .
$$

We know that under some natural assumptions the Navier-Stokes equation (8) has a weak (Leray-Hopf) solution with a bounded energy norm. Moreover, it is known (see, e.g., (18)) that $u_{t}$ belongs to $L^{5 / 4}$. Hence, we can always select $S_{0}\left(Q_{T}\right)$ such that all integrals entering $\mathbb{M}_{\pi}(v, u)$ are defined provided that $v$ (approximation) has the same (minimal) differentiability properties as $u$. In view of (32), any numerical method which is based (explicitly or implicitly) on minimization of the residual functional (31) approximates $u$ in terms of the measure $\mathbb{M}_{\pi}(v, u)$. If the problem has only one solution $u$, then $\left|\mathcal{L}_{v}\right|>0$ if $v \neq u$ and (32) shows that $\mathbb{M}_{\pi}(v, u)$ vanishes if and only if $v=u$. However, in general uniqueness is not proved and, therefore, we cannot guarantee that this measure of the distance between $v$ and $u$ is a metric.

\section{$3 \quad$ Finding $\Pi_{S_{0}^{1, \alpha}}(v)$ for $\alpha \in(1, \infty)$}

Now we discuss computable bounds of the projection operator $\Pi_{S_{0}^{1, \alpha}}(v)$. In some cases, such a bound is easy to obtain. Let $v$ be an approximate solution $v_{h} \in V_{h}$ defined of a certain mesh $\mathcal{T}_{h}$. Assume that we have a suitable postprocessing operator $P_{h}: V_{h} \rightarrow S_{0}^{1,2}\left(\Omega, \mathbb{R}^{d}\right)$. In view of (14), we can set

$$
\Pi_{S_{0}^{1,2}}\left(v_{h}\right):=\left\|\nabla\left(v_{h}-P_{h} v_{h}\right)\right\|
$$

For some finite element approximations in 2D, such type operators can be constructed by means of stream functions and equilibrated approximations. Then, the estimates (26), (28), and (30) can be directly used. However, in other cases such type methods based on explicit reconstruction of a divergence free field may be inefficient and/or too complicated. Below we discuss another method and deduce estimates of the distance between a function $\widehat{v} \in W_{0}^{1, \alpha}\left(\Omega, \mathbb{R}^{d}\right)$ and the respective subspace $S_{0}^{1, \alpha}\left(\Omega, \mathbb{R}^{d}\right)$ containing divergence free fields. We begin with the most simple (and most interesting) case $\alpha=2$. 
3.1 Finding $\Pi_{S_{0}^{1,2}}(v)$ by the $L B B$ condition

We can deduce $\Pi_{S_{0}^{1,2}}(v)$ using the inf-sup (or LBB) condition (see I. Babuška (1) and F. Brezzi (7)), which reads: there exists a positive constant $c_{\Omega}$ such that

$$
\inf _{\substack{\phi \in \widetilde{L}^{2}(\Omega) \\ \phi \neq 0}} \sup _{\substack{w \in V_{0} \\ w \neq 0}} \frac{\int_{\Omega} \phi \operatorname{div} w d x}{\|\phi\|\|v\|} \geq c_{\Omega},
$$

where $V_{0}:=W_{0}^{1,2}\left(\Omega, \mathbb{R}^{d}\right)$. This condition was introduced, proved, and used in order to justify stability of the Stokes problem and to guarantee convergence of approximations (e.g., see (8) for a consequent discussion). Also, (33) can be justified due to the J. Nečas inequality (see (22) and (6)). Conditions analogous to (33) for various pairs of finite dimensional spaces are necessary to prove stability and convergence of numerical methods developed for viscous incompressible fluids (e.g., see $(8 ; 11 ; 15 ; 20 ; 35)$ ).

It is not difficult to show that (33) yields an upper bound of $\Pi_{S_{0}^{1,2}}(v)$ (see also (26)). Notice that

$$
\begin{aligned}
\inf _{v \in S_{0}^{1,2}} \frac{1}{2}\|\nabla(\stackrel{\circ}{v}-v)\|^{2} & =\inf _{w_{0} \in V_{0}} \sup _{\phi \in \widetilde{L}^{2}(\Omega)} \int_{\Omega}\left(\frac{1}{2}\left\|\nabla\left(w_{0}-v\right)\right\|^{2}-\phi \operatorname{div} w_{0}\right) d x \\
& =\sup _{\phi \in \widetilde{L}^{2}(\Omega)}\left[\inf _{w_{0} \in V_{0}} \int_{\Omega}\left(\frac{1}{2}\left|\nabla w_{0}\right|^{2}-\phi \operatorname{div}\left(w_{0}+v\right)\right) d x\right] .
\end{aligned}
$$

Consider the term in the square brackets. Let $\bar{w}_{0}$ be a nonzero element of the space $V_{0}$. Obviously, $t \bar{w}_{0} \in V_{0}$ for any $t \in \mathbb{R}$. Therefore,

$$
\begin{aligned}
\inf _{w_{0} \in V_{0}} \int_{\Omega}\left(\frac{1}{2}\left|\nabla w_{0}\right|^{2}-\right. & \left.\phi \operatorname{div}\left(w_{0}+v\right)\right) d x \leq \\
\leq & \frac{1}{2} t^{2}\left\|\nabla \bar{w}_{0}\right\|^{2}-t \int_{\Omega} \phi \operatorname{div} \bar{w}_{0} d x-\int_{\Omega} \phi \operatorname{div} v d x
\end{aligned}
$$

Setting $t=t^{*}:=\left(\int_{\Omega} \phi \operatorname{div} \bar{w}_{0} d x\right)\left\|\nabla \bar{w}_{0}\right\|^{-2}$, we minimize the right-hand side of (34) and find that

$$
\inf _{w_{0} \in V_{0}} \int_{\Omega}\left(\frac{1}{2}\left|\nabla w_{0}\right|^{2}-\phi \operatorname{div}\left(w_{0}+v\right)\right) d x \leq-\frac{\left(\int_{\Omega} \phi \operatorname{div} \bar{w}_{0} d x\right)^{2}}{2\left\|\nabla \bar{w}_{0}\right\|^{2}}-\int_{\Omega} \phi \operatorname{div} v d x .
$$

Since $\bar{w}_{0}$ is an arbitrary function, we can take supremum over $\bar{w}_{0}$ in the right 
hand side. In view of (33),

$$
-\sup _{\substack{\bar{w}_{0} \in V_{0} \\ \bar{w}_{0} \neq 0}} \frac{\int_{\Omega} \phi \operatorname{div} \bar{w}_{0} d x}{\left\|\nabla \bar{w}_{0}\right\|} \leq-c_{\Omega}\|\phi\| \quad \forall \phi \in \widetilde{L}(\Omega) .
$$

Hence

$$
\begin{array}{r}
\inf _{w_{0} \in V_{0}} \int_{\Omega}\left(\frac{1}{2}\left|\nabla w_{0}\right|^{2}-\phi \operatorname{div}\left(w_{0}+v\right)\right) d x \leq-\frac{c_{\Omega}^{2}}{2}\|\phi\|^{2}-\int_{\Omega} \phi \operatorname{div} v d x \leq \\
\leq-\frac{c_{\Omega}^{2}}{2}\|\phi\|^{2}+\|\phi\|\|\operatorname{div} v\| .
\end{array}
$$

We take supremum over $\phi$ and conclude that

$$
\inf _{v \in S_{0}^{1,2}}\|\nabla(v-\stackrel{\circ}{v})\| \leq c_{\Omega}^{-1}\|\operatorname{div} v\|=: \Pi_{S_{0}^{1,2}}(v)
$$

\subsection{Finding $\Pi_{S_{0}^{1, \alpha}}(v)$ by the stability theorem}

A general method of finding $\Pi_{S_{0}^{1,2}}(v)$ follows Theorem 2 (which was proved by I. Babuška and A. K. Aziz (2) for $d=2$ and by O. A. Ladyzhenskaya and V. A. Solonnikov $(19 ; 33)$ for $d \geq 2)$.

Theorem 2 For any $f \in L^{2}(\Omega)$ with zero mean $\{f\}_{\Omega}$, there exists a function $w_{f} \in W_{0}^{1,2}\left(\Omega, \mathbb{R}^{d}\right)$ such that

$$
\operatorname{div} w_{f}=f \quad \text { and } \quad\left\|\nabla w_{f}\right\| \leq \kappa_{\Omega}\|f\|,
$$

where $\kappa_{\Omega}$ is a positive constant depending on $\Omega$.

It is easy to see that (36) yields the inf-sup condition (33) with $c_{\Omega}=\left(\kappa_{\Omega}\right)^{-1}$.

Extension of Theorem 2 to $L^{\alpha}$ spaces is presented in M. Bogovskii (5) and K. Piletskas (25).

Theorem 3 Let $f \in L^{\alpha}(\Omega)$ and $1<\alpha<+\infty$. If $\{f\}_{\Omega}=0$, then there exists $v_{f} \in W_{0}^{1, \alpha}\left(\Omega, \mathbb{R}^{d}\right)$ such that

$$
\operatorname{div} v_{f}=f \quad \text { and } \quad\left\|\nabla v_{f}\right\|_{\alpha, \Omega} \leq \kappa_{\Omega, \alpha}\left\|\operatorname{div} v_{f}\right\|_{\alpha, \Omega},
$$

where $\kappa_{\Omega, \alpha}$ is a positive constant depending on $\alpha$ and $\Omega$.

Theorems 2 and 3 imply the following result. 
Lemma 1 For any $v_{0} \in W_{0}^{1, \alpha}\left(\Omega, \mathbb{R}^{d}\right)$

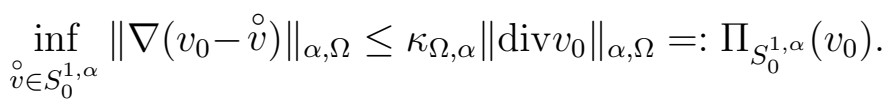

Proof. Let $v_{0}$ be a function in $W_{0}^{1, \alpha}\left(\Omega, \mathbb{R}^{d}\right)$. Since $\left\{\operatorname{div} v_{0}\right\}_{\Omega}=0$, we can find $v_{f} \in W_{0}^{1, \alpha}\left(\Omega, \mathbb{R}^{d}\right)$ such that

$$
\operatorname{div} v_{f}=f:=\operatorname{div} v_{0} .
$$

Due to (37), the function $\stackrel{\circ}{v}:=v-v_{f}$ belongs to $S_{0}^{1, \alpha}\left(\Omega, \mathbb{R}^{d}\right)$ and

$$
\left\|\nabla\left(v_{0}-\stackrel{\circ}{v}\right)\right\|_{\alpha, \Omega}=\left\|\nabla v_{f}\right\|_{\alpha, \Omega} \leq \kappa_{\Omega, \alpha}\left\|\operatorname{div} v_{0}\right\|_{\alpha, \Omega} .
$$

Thus, we arrive at the estimate (38).

Lemma 1 shows that the distance between $v \in W_{0}^{1, \alpha}\left(\Omega, \mathbb{R}^{d}\right)$ and the set of divergence free fields is easy to estimate from above provided that the constant $\kappa_{\Omega, \alpha}$ (or a suitable upper bound of it) is known. However, finding $\kappa_{\Omega, \alpha}$ may be a very difficult problem. To the best of our knowledge, estimates of the constant are known mainly for the case $\alpha=2$ and $d=2$ (see $(34 ; 23 ; 10)$ ). Interesting results that probably can essentially improve all previously known estimates has been recently obtained in (9) (these estimates are valid for bounded domains in $\mathbb{R}^{2}$ which are star-shaped with respect to a ball). In (24), an estimate was obtained for domains in $\mathbb{R}^{3}$ that satisfy some extra regularity assumptions. However, for complicated domains with Lipschitz boundaries analogous estimates are unknown.

Numerical evaluation of the constant (based on minimization of the respective quotient) could be a possible way out provided that the minimiser is sufficiently regular. Regrettably minimising sequences often converge to highly singular functions. On Figure 1 we depict approximations of the extremal function in the Nečas inequality (which is a form of (33))

$$
\|p\| \leq \kappa_{\Omega}\|\nabla p\|_{-1, \Omega} \quad p \in \widetilde{L}^{2}(\Omega),
$$

computed by means of the Fourier series with 8, 36, and 120 terms for the square domain $\Omega=(-\pi, \pi) \times(-\pi, \pi)$. It is easy to see that the minimizer has a clear tendency to form singular components near the corners (in (17) the minimiser is depicted with singularities pointed out in opposite directions at opposite corners). The respective values of the constant tend to a quantity close to 0.5 ; there is a hypothesis (9) that the exact value of the constant is very close or equal to $\left(\frac{1}{2}-\frac{1}{\pi}\right)^{1 / 2}$.

A collection of approximate values of $\kappa_{\Omega}$ for $\alpha=2$ and various domaines (mainly in $\mathbb{R}^{2}$ ) is presented in (17). They are computed using sufficiently rich 
finite element subspaces and probably provide rather sharp estimates, but strictly speaking we cannot say that they are fully guaranteed.
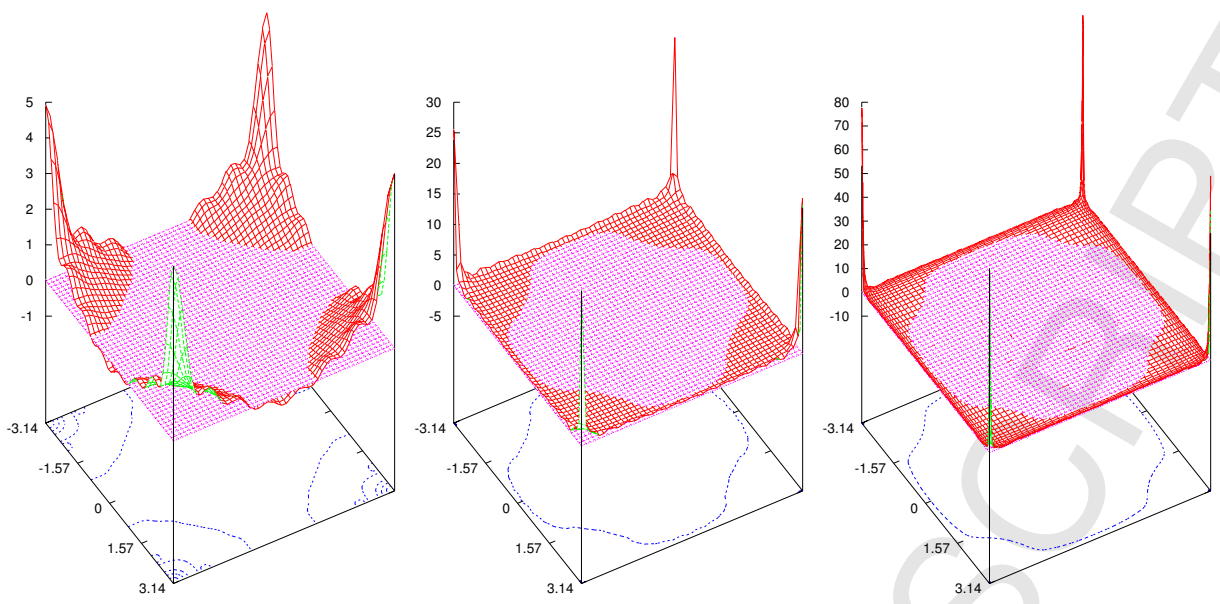

Fig. 1. Extremal function in (39) for 8, 36, and 120 terms

Summarising, we can say that guaranteed estimates of $\kappa_{\Omega, 2}$ can be found by analytical methods for special classes of 2D domains. On the other hand, so far there is no reliable numerical technology for computing guaranteed and sufficiently sharp two-sided bounds of these constants for Lipschitz domains if $d>2$.

A way to bypass these difficulties and obtain computable estimates of the distance to divergence free fields is discussed in the next section. It is based on the idea to use constants $\kappa_{\Omega_{i}, \alpha}$ for a collection of relatively simple subdomains, which union is $\Omega$.

\section{$4 \quad$ Finding $\Pi_{S_{0}^{1, \alpha}}(v)$ by domain decomposition}

First, we present advanced forms of the stability theorem adapted to the case where $\Omega$ is divided into a collection of non-overlapping Lipschitz subdomains $\Omega_{i}, i=1,2, \ldots N$.

Theorem 4 Let $f \in L^{\alpha}(\Omega)$ and $\{f\}_{\Omega_{i}}=0$ for $i=1,2, \ldots, N$. Then, there exists $v_{f} \in W_{0}^{1, \alpha}\left(\Omega, \mathbb{R}^{d}\right)$ such that 


$$
\operatorname{div} v_{f}=f \quad \text { and } \quad\left\|\nabla v_{f}\right\|_{\alpha, \Omega}^{\alpha} \leq \sum_{i=1}^{N} \kappa_{\Omega_{i}, \alpha}^{\alpha}\|f\|_{\alpha, \Omega}^{\alpha}
$$

where $\kappa_{\Omega_{i}, \alpha}$ are positive constants associated with subdomains $\Omega_{i}$.

Proof. We apply Theorem 3 to $\Omega_{i}$. There exists $v_{f, i} \in W_{0}^{1, \alpha}\left(\Omega_{i}, \mathbb{R}^{d}\right)$ such that

$$
\operatorname{div} v_{f, i}=f \text { in } \Omega_{i} \quad \text { and } \quad\left\|\nabla v_{f, i}\right\|_{\alpha, \Omega_{i}} \leq \kappa_{\Omega_{i}, \alpha}\|f\|_{\alpha, \Omega_{i}} .
$$

Define $v_{f}(x)=v_{f, i}(x)$ if $x \in \Omega_{i}$. The function $v_{f}$ belongs to $W_{0}^{1, \alpha}\left(\Omega_{i}, \mathbb{R}^{d}\right)$. It is easy to see that $\operatorname{div} v_{f}=f$ and

$$
\left\|\nabla v_{f}\right\|_{\alpha, \Omega}^{\alpha}=\sum_{i=1}^{n}\left\|\nabla v_{f, i}\right\|_{\alpha, \Omega_{i}}^{\alpha} \leq \sum_{i=1}^{n} \kappa_{\Omega_{i}, \alpha}^{\alpha}\|f\|_{\alpha, \Omega_{i}}^{\alpha}
$$

Thus, we obtain (40).

Theorem 4 implies an estimate of the distance between $v \in W_{0}^{1, \alpha}\left(\Omega, \mathbb{R}^{d}\right)$ and $S_{0}^{1, \alpha}\left(\Omega, \mathbb{R}^{d}\right)$ provided that $v$ satisfies an additional integral type condition. The idea to use this condition for getting easily computable estimates of the distance to the set of divergence free fields was originally introduced in (32). Assume that $v \in W^{1, \alpha}\left(\Omega, \mathbb{R}^{d}\right)$ and satisfies the following weak solenoidality conditions:

$$
\{\operatorname{div} v\}_{\Omega_{i}}=0 \quad \forall i=1,2, \ldots, N .
$$

If $v$ does not satisfy (41), then the corresponding correction can be done by changing $N$ parameters in the representation of this function. Since

$$
\int_{\Omega_{i}} \operatorname{div} v d x=\int_{\partial \Omega_{i}} v \cdot \mathrm{n}_{i} d s \quad i=1,2, \ldots, N
$$

where $\mathrm{n}_{i}$ is the outward normal to the boundary $\partial \Omega_{i}$, changing the parameters should be done such that all the boundary integrals vanish. Therefore, from the computational point of view integral type conditions (41) do not generate essential difficulties (unlike the point wise condition $\operatorname{div} v=0$ ). Lemma below shows that for the vector functions satisfying (41) we have a simple estimate of the distance to the set of divergence free fields (for $\alpha=2$, the estimate (42) has been earlier established in (30)).

Lemma 2 If $v \in W_{0}^{1, \alpha}\left(\Omega, \mathbb{R}^{d}\right)$ and satisfies (41), then

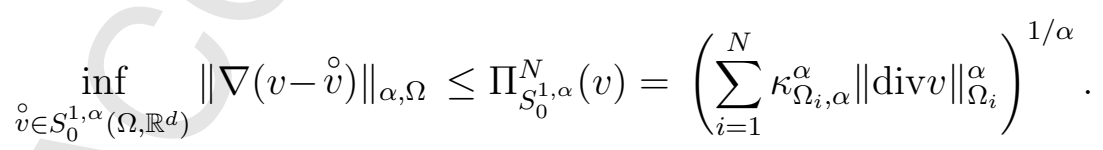


Proof. We set $f=\operatorname{div} v$. Then, there exists $v_{f} \in W_{0}^{1, \alpha}\left(\Omega, \mathbb{R}^{d}\right)$ satisfying (40). The function $v_{0}=v-v_{f}$ is divergence free and

$$
\|\nabla(v-\stackrel{\circ}{v})\|_{\alpha, \Omega}^{\alpha}=\left\|\nabla v_{f}\right\|_{\alpha, \Omega}^{\alpha} \leq \sum_{i=1}^{N} \kappa_{\alpha, \Omega_{i}}^{\alpha}\|\operatorname{div} v\|_{\alpha, \Omega_{i}}^{\alpha}
$$

which implies (42).

Remark 1 By the same arguments we conclude that for any $v \in W^{1, \alpha}\left(\Omega, \mathbb{R}^{d}\right)$ there exists a divergence free field $\stackrel{\circ}{v}$ such that $\stackrel{\circ}{v}=v$ on $\partial \Omega$ and $\|\nabla(v-\stackrel{\circ}{v})\|_{\alpha, \Omega}$ satisfies (42). This estimate could be helpful for problems with mixed boundary conditions.

Remark 2 We can deduce fully computable estimates of the distance, which are valid without the conditions or (41). Let $\mu_{i}:=\int_{\Omega_{i}} \operatorname{div} v_{0} d x$. Usually it is not difficult to find a correcting function $w_{0} \in W_{0}^{1, \alpha}\left(\Omega, \mathbb{R}^{d}\right)$ such that

$$
\int_{\Omega_{i}} \operatorname{div} w_{0} d x=\mu_{i} \quad \text { for } i=1,2, \ldots, N .
$$

On polygonal meshes such a function can be constructed by, e.g., piecewise polynomial vector functions. Then $v_{0}-w_{0}$ satisfies (41) and we have

$$
\inf _{\dot{v} \in S^{1, \alpha}\left(\Omega, \mathbb{R}^{d}\right)}\left\|\nabla\left(v_{0}-\stackrel{\circ}{v}\right)\right\|_{\alpha, \Omega} \leq\left(\sum_{i=1}^{N} \kappa_{\Omega_{i}, \alpha}^{\alpha}\left\|\operatorname{div}\left(v_{0}-w_{0}\right)\right\|_{\alpha, \Omega_{i}}^{\alpha}\right)^{1 / \alpha}+\left\|\nabla w_{0}\right\|_{\alpha, \Omega} .
$$

Since $w_{0} \in W_{0}^{1, \alpha}\left(\Omega, \mathbb{R}^{d}\right)$ can be selected in many different ways, optimal (or close to optimal) estimated should involve a certain minimisation procedure with respect to $w_{0}$. However, if the values $\mu_{i}$ are small (what is natural to await from good approximations), then the overall effect of optimisation may be insignificant.

By using (42) together with (16), we obtain the following error majorant for the Stokes problem:

$$
\mu\|\nabla(u-v)\| \leq\|\tau+q \mathbb{I}-\mu \nabla v\|+C_{F \Omega}\|\operatorname{Div} \tau+f\|+2 \mu\left(\sum_{i=1}^{N} \kappa_{\Omega_{i}}^{2}\|\operatorname{div} v\|_{\Omega_{i}}^{2}\right)^{1 / 2} .
$$

Another localised version of Theorems 2 has been proved in (31) for $\alpha=2$. We use similar arguments and extend it to the case $\alpha \in(1,+\infty)$. Let $\Omega$ be decomposed into a collection of Lipschitz subdomains $D_{k}, k=1,2, \ldots, K$. 
Subdomains $D_{k}$ may overlap. They generate a decomposition of $\Omega$ into a set of nonoverlapping subdomains $\Omega_{i}(k=1,2, \ldots, N)$ so that

$$
\bar{\Omega}=\bigcup_{k=1}^{K} \bar{D}_{k}=\bigcup_{i=1}^{N} \bar{\Omega}_{i}, \quad \Omega_{i} \cap \Omega_{j}=\emptyset \text { for } i \neq j
$$

and the intersection of $D_{k}$ and $D_{l}$ is either empty or consists of one or several subdomains $\Omega_{i}$ (any $\Omega_{i}$ is contained in one or several $D_{k}$ ).

Theorem 5 Let $f \in L^{\alpha}(\Omega)$ be such that $\{f\}_{\Omega_{i}}=0$ for $i=1,2, \ldots, N$. Then, there exists a function $v_{f} \in W_{0}^{1, \alpha}\left(\Omega, \mathbb{R}^{d}\right)$ such that $\operatorname{div} v_{f}=f$ in $\Omega$ and

$$
\left\|\nabla v_{f}\right\|_{\Omega} \leq \sum_{i=1}^{N} \kappa_{i}\|f\|_{\Omega_{i}}
$$

where

$$
\kappa_{i}=\inf _{k=1, \ldots, K} \rho_{k}, \quad \rho_{k}= \begin{cases}\kappa_{D_{k}, \alpha} & \text { if } \Omega_{i} \subset D_{k} \\ +\infty & \text { if } \Omega_{i} \not \subset D_{k}\end{cases}
$$

and $\kappa_{D_{k}, \alpha}$ denote the respective constants associated with subdomains.

Proof. It is easy to see that $f=\sum_{i=1}^{N} f_{i}$, where

$$
f_{i}(x)= \begin{cases}f & \text { if } x \in \Omega_{i} \\ 0 & \text { if } x \notin \Omega_{i}\end{cases}
$$

There exists at least one $D_{k}$ such that $\Omega_{i} \subset D_{k}$. If there are several $D_{k}$ containing $\Omega_{i}$, then we select $D_{k}$ with minimal $\kappa_{D_{k}, \alpha}$ (see (44)). Since $\left\{f_{i}\right\}_{D_{k}}=0$, and $D_{k}$ is a Lipschitz domain, we can find $v_{f_{i}} \in W_{0}^{1, \alpha}\left(D_{k}, \mathbb{R}^{d}\right)$ such that

$$
\operatorname{div} v_{f_{i}}=f_{i} \quad \text { in } D_{k}
$$

and

$$
\left\|\nabla v_{f_{i}}\right\|_{\alpha, D_{k}} \leq \kappa_{i}\left\|f_{i}\right\|_{\alpha, D_{k}}=\kappa_{i}\|f\|_{\alpha, \Omega_{i}} .
$$

We extend $v_{f_{i}}$ by zero to $\Omega \backslash D_{k}$ and find that (45) holds in $\Omega$. Moreover,

$$
\left\|\nabla v_{f_{i}}\right\|_{\alpha, \Omega} \leq \kappa_{i}\|f\|_{\alpha, \Omega_{i}}
$$

Set $v_{f}=\sum_{i=1}^{N} v_{f_{i}} \in W_{0}^{1, \alpha}\left(\Omega, \mathbb{R}^{d}\right)$. Then $\operatorname{div} v_{f}=f$. Since

$$
\left\|\nabla v_{f}\right\|_{\alpha, \Omega} \leq \sum_{i=1}^{n}\left\|\nabla v_{f_{i}}\right\|_{\alpha, D_{k}} \leq \sum_{i=1}^{N} \kappa_{i}\left\|f_{i}\right\|_{\alpha, D_{k}}=\sum_{i=1}^{N} \kappa_{i}\|f\|_{\alpha, \Omega_{i}}
$$

we arrive at (43). 
Theorem 5 implies another estimate of the distance to the set of divergence free fields.

Lemma 3 Assume $v_{0} \in W_{0}^{1, \alpha}\left(\Omega, \mathbb{R}^{d}\right)$ satisfies (41). Then,

$$
\inf _{\stackrel{\circ}{v} \in S^{1, \alpha}\left(\Omega, \mathbb{R}^{d}\right)}\left\|\nabla\left(v_{0}-\stackrel{\circ}{v}\right)\right\|_{\alpha, \Omega} \leq \Pi_{S_{0}^{1, \alpha}}^{N, K}\left(v_{0}\right)=\sum_{i=1}^{N} \kappa_{i}\left\|\operatorname{div} v_{0}\right\|_{\alpha, \Omega_{i}},
$$

where $\kappa_{i}$ are defined by (44).

Remark 3 For problems with mixed boundary conditions (where the Dirichlet boundary conditions are set on $\Gamma_{D} \subset \partial \Omega$ ) it is important to have majorants of the distance between a function

$$
v \in W_{0, \Gamma_{D}}^{1, \alpha}\left(\Omega, \mathbb{R}^{d}\right):=\left\{v \in W^{1, \alpha}\left(\Omega, \mathbb{R}^{d}\right) \mid v=0 \text { on } \Gamma_{D}\right\},
$$

and $S_{0, \Gamma_{D}}^{1, \alpha}\left(\Omega, \mathbb{R}^{d}\right)=\left\{v \in W_{0, \Gamma_{D}}^{1, \alpha}\left(\Omega, \mathbb{R}^{d}\right) \mid \operatorname{div} v=0\right\}$. This question is considered in (31). In particular, for $\alpha=2$ it is shown that

$$
\inf _{\substack{v \in S_{0, \Gamma_{D}}^{1,2}\left(\Omega, \mathbb{R}^{d}\right)}}\|\nabla(v-\stackrel{\circ}{v})\| \leq \kappa_{\Omega}\|\operatorname{div} v\|+C_{*}\left|\int_{\Omega} \operatorname{div} v d x\right|,
$$

where

$$
C_{*}=\frac{1}{\left\|\nabla u_{*}\right\|}\left(\kappa_{\Omega} \frac{\left\|\operatorname{div} u_{*}\right\|}{\left\|\nabla u_{*}\right\|}+1\right),
$$

and $u_{*}$ solves a specially constructed auxiliary boundary value problem.

Acknowledgment. The research was partially supported by RFBR grant N 14-01-00162. Also, the author thanks SPOMECH project CZ.1.07/2.3.00/20.0070 and exchange visiting program of the Czech Academy of Sciences.

\section{References}

[1] I. Babuška, The finite element method with Lagrangian multipliers, Numer. Math. 20 (1973) 179-192.

[2] I. Babuška, A. K. Aziz, Survey Lectures on the Mathematical Foundations of the Finite Element Method, Academic Press, New York, 1972.

[3] A. Bermúdez, R. Durán, R. Rodríguez, Finite element analysis of compressible and incompressible fluid-solid systems, Math. Comput. 67 (1998) 111-136.

[4] P. Bochev, M. Gunzburger, Finite element methods of least-squares type, SIAM Review 40 (1998) 789-837. 
[5] M. E. Bogovskii, Solution of the first boundary value problem for the equation of continuity of an incompressible medium, Soviet Math. Dokl. 248 (1979) 1037-1040.

[6] J. Bramble, A proof of the inf-sup condition for the Stokes equations on Lipschitz domains, Math. Models Methods Appl. Sci. 13 (2003) 361-371.

[7] F. Brezzi, On the existence, uniqueness and approximation of saddlepoint problems arising from Lagrange multipliers, R.A.I.R.O., Anal. Numer. R2(1974) 129-151.

[8] F. Brezzi, M. Fortin, Mixed and Hybrid Finite Element Methods, Springer Series in Computational Mathematics, 15, New York, 1991.

[9] M. Costabel and M. Dauge. On the inequalities of Babuška-Aziz, Friedrichs and Horgan-Payne, Archive for Rational Mechanics and Analysis 217 (2015) 873-898 (arXiv 1303.6141v1, 2013).

[10] M. Dobrowolski, On the LBB constant on stretched domains, Math. Nachr. 254-255 (2003) 64-67.

[11] M. Feistauer, Mathematical Methods in Fluid Dynamics, Longman, Harlow, 1993.

[12] M. Fuchs, S. Repin, Estimates for the deviation from the exact solutions of variational problems modeling certain classes of generalized Newtonian fluids, Math. Methods Appl. Sci. 29 (2010), 2225-2244.

[13] M. Fuchs, S. Repin, Estimates of the deviations from the exact solutions for variational inequalities describing the stationary flow of certain viscous incompressible fluids, Math. Methods Appl. Sci. 33 (2010) 1136-1147.

[14] G. P. Galdi. An Introduction to the Mathematical Theory of the NavierStokes Equations, Springer, New York, 1994.

[15] V. Girault and P. A. Raviart. Finite Element Approximation of the Navier-Stokes Equations, Springer, Berlin, 1986.

[16] J. G. Heywood, W. Nagata, W. Xie, A numerically based existence theorem for the Navier-Stokes equations J. Math. Fluid Mech. 1 (1999) 5-23.

[17] M. Kessler. Die Ladyzhenskaya-Konstante in der numerischen Behandlung von Stromungsproblemen Dissertation zur Erlangung des natur- wissenschaftlichen. Doktorgrades der Bayerischen Julius-Maximilians- Universität Würzburg, 2000.

[18] O. A. Ladyzhenskaya. Mathematical Problems in the Dynamics of a Viscous Incompressible Flow, second ed., Gordon and Breach, New York, 1969.

[19] O. A. Ladyzenskaja, V. A. Solonnikov, Some problems of vector analysis, and generalized formulations of boundary value problems for the NavierStokes equation, Zap. Nauchn. Sem. Leningrad. Otdel. Mat. Inst. Steklov. 59 (1976), 81-116 (Russian).

[20] D. S. Malkus, Eigenproblems associated with the discrete LBB-condition for incompressible finite elements, Int. J. Engrg. Sci. 19 (1981) 1299-1310.

[21] A. Mikhailov, S. Repin, Estimates of deviations from exact solution of the Stokes problem in the velocity-vorticity-pressure formulation, Zap. Nauchn. Sem. S.-Peterburg. Otdel. Mat. Inst. Steklov. 397 (2011) 73-87. 
[22] J. Nečas, Les Méthodes Directes en Théorie des Équations Elliptiques, Masson et Cie, Éditeurs, Paris; Academia, Éditeurs, Prague 1967.

[23] M.A. Olshanskii, E.V. Chizhonkov, On the best constant in the inf sup condition for prolonged rectangular domains, Mat. Zametki 67 (2000) 387-396 (Russian).

[24] L. E. Payne, A bound for the optimal constant in an inequality of Ladyzhenskaya and Solonnikov, IMA J. Appl. Math. 72 (2007), 563-569.

[25] K. I. Piletskas, On spaces of solenoidal vectors, Zap. Nauchn. Sem. Leningrad. Otdel. Mat. Inst. Steklov. 96 (1980) 237-239.

[26] S. Repin, A posteriori estimates for the Stokes problem, J. Math. Sci. (New York) 109 (2002) 1950-1964.

[27] S. Repin, Estimates of deviations from exact solutions for some boundary-value problems with incompressibility condition, St. Petersburg Math. J., 16 (2004) 124-161.

[28] S. Repin, A Posteriori Estimates for Partial Differential Equations, Walter de Gruyter, Berlin, 2008.

[29] S. Repin, On measures of errors for nonlinear variational problems, Russian J. Numer. Anal. Math. Modelling, 27 (2012) 577- 584.

[30] S. Repin, Estimates of deviations from exact solution of the generalized Oseen problem, Zap. Nauchn. Sem. S.-Peterburg. Otdel. Mat. Inst. Steklov. 410 (2013) 110-130

[31] S. Repin, Estimates of the distance to the set of divergence free fields, Zap. Nauchn. Sem. S.-Peterburg. Otdel. Mat. Inst. Steklov. 425 (2014) 99-116.

[32] S. Repin, R. Stenberg, A posteriori error estimates for the generalized Stokes problem, J. Math. Sci. (New York), 142 (2007) 1828-1843.

[33] V. A. Solonnikov, Estimates for solutions of a non-stationary linearized system of Navier- Stokes equations, Zap. Nauchn. Sem. leningrad. Otdel. Mat. Inst. Steklov. 70 (1964) 213-317.

[34] G. Stoyan, Towards discrete Velte decompositions and narrow bounds for Inf-Sup constants, Comput. Math. Appl. 38 (1999) 243-261.

[35] R. Temam. Navier-Stokes Equations. Theory and Numerical Analysis, Studies in Mathematics and its Applications, 2, North-Holland, Amsterdam, 1979.

[36] J. Valdman, Minimization of functional majorant in a posteriori error analysis based on $H$ (div) multigrid-preconditioned CG method, Adv. Numer. Anal. (2009), Art. ID 164519. 